\title{
Increments in DNA-thioguanine level during thiopurine-enhanced maintenance therapy of acute lymphoblastic leukemia
}

Haematologica 2021

Volume 106(11)2824-2833

\section{Correspondence:}

KJELD SCHMIEGELOW

kschmiegelow@rh.dk

Received: December 14, 2020.

Accepted: April 28, 2021.

Pre-published: May 27, 2021.

https://doi.org/10.3324/haematol.2020.278166

(C)2021 Ferrata Storti Foundation

Material published in Haematologica is covered by copyright. All rights are reserved to the Ferrata Storti Foundation. Use of published material is allowed under the following terms and conditions:

https://creativecommons.org/licenses/by-nc/4.0/legalcode. Copies of published material are allowed for personal or internal use. Sharing published material for non-commercial purposes is subject to the following conditions:

https://creativecommons.org/licenses/by-nc/4.0/legalcode, sect. 3. Reproducing and sharing published material for commercial purposes is not allowed without permission in writing from the publisher.

\author{
Rikke Hebo Larsen, ${ }^{1}$ Cecilie Utke Rank, ${ }^{1,2}$ Kathrine Grell, ${ }^{1,3}$ Lisbeth Nørgaard \\ Møller, ${ }^{1}$ Ulrik Malthe Overgaard, ${ }^{2}$ Peter Kampmann, ${ }^{2}$ Jacob Nersting, ${ }^{1}$ Matilda \\ Degn, ${ }^{1}$ Stine Nygaard Nielsen, ${ }^{1}$ Helle Holst, ${ }^{1}$ Birgitte Klug Albertsen, ${ }^{4,5}$ Peder Skov \\ Wehner, ${ }^{6}$ Michael Thude Callesen, ${ }^{6}$ Jukka Kanerva, ${ }^{7}$ Thomas Leth Frandsen, ${ }^{1}$ \\ Bodil Als-Nielsen, ${ }^{1}$ Lisa Lyngsie Hjalgrim ${ }^{1}$ and Kjeld Schmiegelow ${ }^{1,8}$
}

${ }^{1}$ Department of Pediatrics and Adolescent Medicine, Rigshospitalet, University of Copenhagen, Copenhagen, Denmark; ${ }^{2}$ Department of Hematology, Rigshospitalet, University of Copenhagen, Copenhagen, Denmark; ${ }^{3}$ Section of Biostatistics, Department of Public Health, University of Copenhagen, Copenhagen, Denmark; ${ }^{4}$ Department of Pediatrics and Adolescent Medicine, Aarhus University, Aarhus, Denmark; ${ }^{5}$ Department of Clinical Medicine, Aarhus University, Aarhus, Denmark; ${ }^{6}$ Department of Pediatric Hematology and Oncology, H.C. Andersen Children's Hospital, Odense University Hospital, Odense, Denmark; 'HUS, Helsinki University Hospital, University of Helsinki, New Children's Hospital, Helsinki, Finland and ${ }^{8}$ Institute of Clinical Medicine, Faculty of Medicine, University of Copenhagen, Copenhagen, Denmark

\section{ABSTRACT}

M aintenance therapy containing methotrexate and 6-mercaptopurine is essential to cure acute lymphoblastic leukemia (ALL). Cytotoxicity is elicited by incorporation of thioguanine nucleotides into DNA (DNA-TG), and higher leukocyte DNA-TG is associated with increased relapse-free survival. As 6-thioguanine provides 6fold higher cytosolic levels of thioguanine nucleotides than does 6mercaptopurine, we added low-dose 6-thioguanine to methotrexate/6mercaptopurine maintenance therapy to explore if this combination results in significantly higher DNA-TG. The target population of the "Thiopurine Enhanced ALL Maintenance therapy" (TEAM) study was 30 patients with non-high-risk ALL, aged 1-45 years on methotrexate/6-mercaptopurine maintenance therapy receiving no other systemic chemotherapy. Incremental doses of 6-thioguanine were added to methotrexate/6-mercaptopurine maintenance therapy (starting 6-thioguanine dose: $2.5 \mathrm{mg} / \mathrm{m}^{2} /$ day, maximum: $12.5 \mathrm{mg} / \mathrm{m}^{2} /$ day). The primary endpoint was DNA-TG increments. Thirty-four patients were included, and 30 patients completed maintenance therapy according to the TEAM strategy. Of these 30 patients, $26(87 \%)$ tolerated $10.0-12.5 \mathrm{mg} / \mathrm{m}^{2} /$ day as the maximum 6-thioguanine dose. TEAM resulted in significantly higher DNA-TG levels compared to those in both TEAM patients before their inclusion in TEAM (on average $251 \mathrm{fmol} / \mu \mathrm{g}$ DNA higher [95\% confidence interval: 160-341; $P<0.0001]$ ), and with historical patients receiving standard methotrexate/6-mercaptopurine maintenance therapy (on average $272 \mathrm{fmol} / \mu \mathrm{g}$ DNA higher [95\% confidence interval: 147-398; $P<0.0001])$. TEAM did not increase myelotoxicity or hepatotoxicity. In conclusion, TEAM is an innovative and feasible approach to improve maintenance therapy and results in higher DNA-TG levels without inducing additional toxicity. It may therefore be an effective strategy to reduce the risk of ALL relapse through increased DNA-TG. This will be tested in a randomized ALLTogether-1 substudy.

\section{Introduction}

Overall survival of patients with acute lymphoblastic leukemia (ALL) has improved immensely and now surpasses $90 \%$ in children and $70 \%$ in adults. ${ }^{1.4}$ However, cure rates after relapse have remained poor, and sustained effort to improve first-line ALL therapy further is crucial. ${ }^{5,6}$ Methotrexate/6-mercapto- 
purine based maintenance therapy is considered one of the most important phases of ALL therapy, but with an unmet need for improved treatment strategies. ' Dose adjustments are guided by white blood cell count or absolute neutrophil count, which have been related to relapse rates $^{7-9}$ However, both white blood cell and absolute neutrophil counts show pronounced variation with age, gender, circadian rhythms and ethnicity, and are therefore poor indicators of methotrexate/6-mercaptopurine treatment intensity. ${ }^{10-12}$ Cytotoxicity of 6mercaptopurine is mediated by thioguanine nucleotides, ${ }^{13}$ which mimic guanine nucleotides, and compete with these for incorporation into DNA. Incorporated thioguanine nucleotides (DNA-TG) can, when methylated, mismatch with thymidine (instead of cytosine), which activates the mismatch repair system. Methyl-thioguanine nucleotides will however continue to mismatch and this ultimately leads to cell death due to repetitive but futile activation of the mismatch repair system. ${ }^{13}$ Higher DNATG levels in circulating normal leukocytes during maintenance therapy were recently shown to be associated with reduced relapse rate, ${ }^{14}$ a finding that has subsequently been validated (Toksvang et al. in press). This suggests that DNA-TG levels in normal leukocytes are indicative of pharmacological events in malignant lymphoblasts. Adjustment of maintenance therapy to increase DNA-TG may therefore lead to improved relapse-free survival. However, attempts to obtain higher DNA-TG by solely incrementing methotrexate/6-mercaptopurine doses would generally fail, because of the complex pharmacokinetics of 6-mercaptopurine, and, furthermore, may increase the risk of serious myelotoxicity and hepatotoxicity. ${ }^{13,15-18}$ 6-Thioguanine (6TG) also exerts cytotoxicity through formation of DNA-TG, leading to 6-7 times higher levels of cytosolic thioguanine nucleotides than those derived from 6-mercaptopurine at equipotent doses. ${ }^{16}$ Randomized studies have, however, demonstrated no benefit in overall survival, when 6-mercaptopurine is replaced by 6TG for maintenance therapy. ${ }^{19-22}$ This might reflect the inability of 6TG to generate higher DNA-TG than 6-mercaptopurine as other 6-mercaptopurine metabolites inhibit de novo purine synthesis, which promotes DNA-TG formation by reducing the levels of natural purines to compete with thioguanine nucleotides for DNA incorporation. ${ }^{11,13,14}$ As an alternative, we tested addition of very low 6TG doses to standard methotrexate/6-mercaptopurine maintenance therapy and explored whether this combination could achieve significantly higher DNA-TG levels without inducing additional toxicity.

\section{Methods}

\section{Study population and study design}

Patients aged 1-45 years with non-high-risk ALL (i.e., standard and intermediate risk) treated according to the Nordic Society of Pediatric Hematology and Oncology (NOPHO) ALL2008 proto$\mathrm{col}^{23,24}$ were eligible for the Thiopurine Enhanced ALL Maintenance therapy (TEAM) study.

The TEAM study was designed as a phase I/II non-randomized clinical trial with increments in DNA-TG as the primary efficacy outcome. The DNA-TG levels documented were compared with DNA-TG levels from TEAM patients before TEAM and with historical patients who had received standard methotrexate/6-mer- captopurine maintenance therapy in the ALL2008 maintenance therapy substudy (ALL2008 MT substudy). ${ }^{14}$

Eligible patients were included, when they reached maintenance therapy phase II (maintenance-II) (Online Supplementary Appendix S1). Patients were included during the entire course of maintenance-II but had to have at least 3 months of remaining therapy at the first visit. An exclusion criterion was end-of-induction minimal residual disease-negative bone marrow, because DNA-TG levels are not associated with relapse risk in such patients. ${ }^{14}$ Patients with previous sinusoidal obstruction syndrome were also excluded, because of the association of this syndrome with 6TG at higher doses (Online Supplementary Appendix S1). ${ }^{19}$ The diagnosis of sinusoidal obstruction syndrome was made as defined by Schmiegelow et al. . $^{25}$

The TEAM study was registered at ClinicalTrials.gov: NCT02912676 and granted a EudraCT number: 2014-002248-42. It was approved by the Ethical Committee of the Capital Region of Denmark (H-3-2014-098), and the Danish Medicines Agency (2014-002248-42). The study was conducted according to the Declaration of Helsinki II and Good Clinical Practice guidelines (Online Supplementary Appendix S1).

\section{Maintenance therapy in the NOPHO ALL2008 protocol}

Maintenance therapy in the NOPHO ALL2008 protocol was divided into two phases, maintenance-I and maintenance-II with weekly oral methotrexate and daily oral 6-mercaptopurine constituting the backbone of both phases and targeted to a white blood cell count of $1.5-3.0 \times 10^{9} / \mathrm{L}$. All therapy was discontinued 2.5 years after diagnosis. Patients with standard-risk ALL continued directly from maintenance-I to maintenance-II, whereas patients with intermediate-risk ALL received 6 weeks of delayed intensification before entering maintenance-II. Maintenance-I contained additional systemic chemotherapy as well as intrathecal chemotherapy, and patients with intermediate-risk ALL also received intrathecal chemotherapy during maintenance-II (Online Supplementary Appendix S1).

\section{Outline of the trial therapy}

Incremental doses of 6TG were added to standard methotrexate/6-mercaptopurine maintenance therapy starting at a dose of $2.5 \mathrm{mg} / \mathrm{m}^{2} /$ day and increased by $2.5 \mathrm{mg} / \mathrm{m}^{2} /$ day biweekly until reaching a maximum $6 \mathrm{TG}$ dose of $12.5 \mathrm{mg} / \mathrm{m}^{2} /$ day. The target level of DNA-TG was above $500 \mathrm{fmol} / \mu \mathrm{g}$ DNA (approximate mean DNA-TG at end of methotrexate/6-mercaptopurine-based maintenance therapy ${ }^{14}$ ). TEAM patients had the same white blood cell target of $1.5-3.0 \times 10^{9} / \mathrm{L}$ as all other NOPHO ALL2008 patients. If DNA-TG and/or white blood cell count targets were not reached at the maximum 6TG dose (i.e., $12.5 \mathrm{mg} / \mathrm{m}^{2} /$ day), 6mercaptopurine and/or methotrexate doses were adjusted (Online Supplementary Appendix S1).

\section{Metabolite assessments}

DNA-TG in circulating normal leukocytes, thioguanine nucleotide level in erythrocytes (Ery-TGN) and erythrocyte level of methylated 6-mercaptopurine metabolites (Ery-MeMP) were quantified as previously described by Jacobsen et al. ${ }^{26}$ and Shipkova et al. ${ }^{27}$ (Online Supplementary Appendix S1).

\section{Data analysis and statistics}

For each patient, median values were calculated for all outcome variables. Differences between patients' medians of all outcomes during TEAM therapy and before TEAM followed a normal distribution and were compared using paired Welch $t$-tests.

The patients' medians of outcome variables during TEAM therapy and the historical data from the ALL2008 MT substudy had 
normal distributions, except for Ery-MeMP, and were compared using Welch two-sample t-tests, except for Ery-MeMP which was analyzed with a two-sample Wilcoxon rank sum test.

\section{Results}

\section{Study population}

The target study population of the TEAM study was 30 patients based on a power calculation (provided in Online Supplementary Appendix S2). A total of 34 patients were included in the TEAM study. The patients' characteristics and demographics are summarized in Table 1. Inclusion was completed in December 2018, and the last TEAM patient finished therapy in March 2020. The study was closed for follow-up on August 31, 2020. The TEAM therapy period for data analysis was defined as the time period between 10 weeks after initial 6TG dosing (when patients would have had sufficient time to reach the potential maximum 6TG dose of $12.5 \mathrm{mg} / \mathrm{m}^{2} /$ day) until discontinuation of TEAM therapy. The time period before TEAM was defined as 2 months prior to initiation of 6TG treatment. The historical data from the ALL2008 MT substudy (patients who received standard methotrexate/6-mercaptopurine maintenance therapy) included measurements from 10 weeks after the start of maintenance-II until discontinuation of antileukemic therapy. Of the total 34 patients, 32 patients received TEAM maintenance therapy for more than 10 weeks (Figure 1). Measurements from these 32 patients (denoted TEAM study population) in the period from 10 weeks after initial 6TG dosing until cessation of antileukemic therapy (time period denoted TEAM therapy) are included in analyses comparing outcome variables during TEAM therapy with either before TEAM therapy or historical patients receiving standard methotrexate/6-mercaptopurine maintenance therapy. Two patients discontinued TEAM study participation after having received more than 10 weeks of TEAM maintenance therapy, as described

Table 1. Demographics and patients' characteristics.

\begin{tabular}{lc} 
Characteristic & Value \\
Sex, $\mathrm{n}(\%)$ & \\
Male & $22(65)$ \\
Female & $12(35)$ \\
Age group, $\mathrm{n}(\%)$ & \\
$\quad$ Children (<18 years at diagnosis) & $29(85)$ \\
$\quad$ Adults ( $\geq 18$ years at diagnosis) & $5(15)$ \\
\hline Age (years) at ALL diagnosis & \\
$\quad$ Median (range) & $3(1-34)$ \\
Immunophenotype, $\mathrm{n}(\%)$ & $31(91)$ \\
$\quad$ Precursor B-cell & $3(9)$ \\
$\quad$ T-cell & \\
WBC at ALL diagnosis, x10\%/L & \\
$\quad$ Mean (range) & $30.4(1.6-317)$ \\
Risk group stratification (day 79$), \mathrm{n}(\%)$ & $16(47)$ \\
$\quad$ Standard risk & $18(53)$ \\
$\quad$ Intermediate risk &
\end{tabular}

ALL: acute lymphoblastic leukemia;WBC: white blood cell count.

\section{Included in the TEAM study: \\ 34 patients}

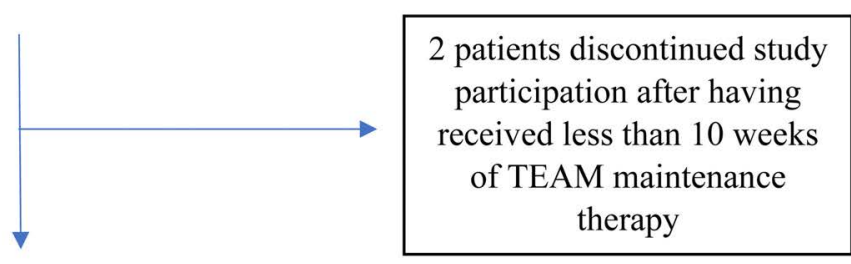

\section{2 patients received more than 10 weeks of TEAM maintenance therapy, i.e. TEAM study population}

30 patients completed ALL therapy according to TEAM strategy

2 patients discontinued study
participation after having
received more than 10 weeks
of TEAM maintenance
therapy

Figure 1. Trial profile. Thirty-two patients received more than 10 weeks of TEAM maintenance therapy, and these patients constitute the TEAM study population. Data from these patients were used for comparison of outcome variables with historical patients (who received standard methotrexate/6-mercaptopurine maintenance therapy), and with TEAM patients before TEAM inclusion. The backgrounds for discontinuation of study participation ( $n=4$ patients) are described in the manuscript. TEAM: thiopurine-enhanced ALL maintenance therapy. 
in "Cessation of TEAM participation" below. The trial profile is illustrated in Figure 1.

Of the total 34 patients, four patients discontinued study participation (see "Cessation of TEAM participation"). The remaining 30 patients completed maintenance-II according to the TEAM strategy. Of these, five patients received 3-6 months of TEAM maintenance therapy (from initiation of 6TG therapy until discontinuation of antileukemic therapy), 17 patients received 7-12 months, and eight patients received more than 12 months of TEAM maintenance therapy. A median of 27 DNA-TG measurements were available per patient (range, 8-45) in the period from initiation of $6 \mathrm{TG}$ until discontinuation of all therapy with an average of 1.8 samples (range, $0.7-3.0$ ) per month.

Of the 34 included patients, 32 were TPMT wild-type, when tested for the G460A and A719G variants, while two patients were heterozygous with one low activity TPMT variant.

The median follow-up for all patients (from discontinuation of antileukemic therapy until August 31, 2020) was 20.9 months (interquartile range [IOR]: 14.4-29.3).

\section{DNA-thioguaninine nucleotide levels}

A total of 645 DNA-TG measurements taken during TEAM therapy were available for analysis. The mean of the patients' DNA-TG medians during TEAM therapy was 764 fmol/ug DNA (IOR, 577-890) and the patients' medians during TEAM therapy varied 5-fold (Figure 2). DNA-TG medians from TEAM patients before their inclusion in the TEAM protocol are presented in Online Supplementary Appendix S2 and Online Supplementary Figure S1.

DNA-TG levels (i.e., patients' medians) were on average $272 \mathrm{fmol} / \mu \mathrm{g}$ DNA higher during TEAM therapy (95\% confidence interval $[95 \% \mathrm{CI}$ : 147-398; $P<0.0001)$ than historical data from the ALL2008 MT substudy, in which patients received standard methotrexate/6-mercaptopurine maintenance therapy (Figure 3; Tables 2 and 3) ${ }^{14}$ If these results are entered into the regression model from the ALL2008 MT substudy concerning estimation of reduction in relapse haz- ard rate with increasing DNA-TG levels, ${ }^{14}$ the DNA-TG increment with the TEAM strategy would have corresponded to a $59 \%$ reduction in the hazard rate of relapse (1$\left[0.72^{2.72}\right]=59 \%$ ). Furthermore, the average of DNA-TG measurements at the end of maintenance-II was approximately $500 \mathrm{fmol} / \mu \mathrm{g}$ DNA in the ALL2008 MT substudy, ${ }^{14}$ whereas TEAM patients had an average DNA-TG level of $933 \mathrm{fmol} / \mu \mathrm{g}$ DNA in the last month of maintenance-II. The number of patients and samples for the various outcomes from the ALL2008 MT substudy are provided in Online Supplementary Appendix S2.

DNA-TG levels during TEAM therapy were on average $251 \mathrm{fmol} / \mu \mathrm{g}$ DNA higher (95\% CI: 160-341; $P<0.0001)$ than those in TEAM patients before participation in the TEAM study. Of the 32 patients who received more than 10 weeks of TEAM maintenance therapy, 27 patients obtained the DNA-TG target above $500 \mathrm{fmol} / \mu \mathrm{g}$ DNA during TEAM therapy. Five patients did not reach the target DNA-TG level. A detailed description of these patients is provided in Online Supplementary Appendix S2.

During the entire course of the TEAM study, two patients experienced an episode of three consecutive measurements of DNA-TG above $1500 \mathrm{fmol} / \mathrm{\mu g}$ DNA, which is greater than the $99^{\text {th }}$ percentile of the ALL2008 MT substudy DNATG distribution, ${ }^{14}$ without excessive myelotoxicity or hepatotoxicity.

\section{Toxicities}

Treatment according to the TEAM strategy did not lead to increased myelotoxicity or hepatotoxicity, when compared with data from the ALL2008 MT substudy or when compared with data before TEAM.

\section{Myelosuppression}

No significant differences were observed when white blood cell count, absolute neutrophil count and hemoglobin level were compared between the period of TEAM therapy and before TEAM $(P=0.78, P=0.40$, and $P=0.10$, respectively) (Figure 4A, B; Tables 2 and 3). No serious infections were

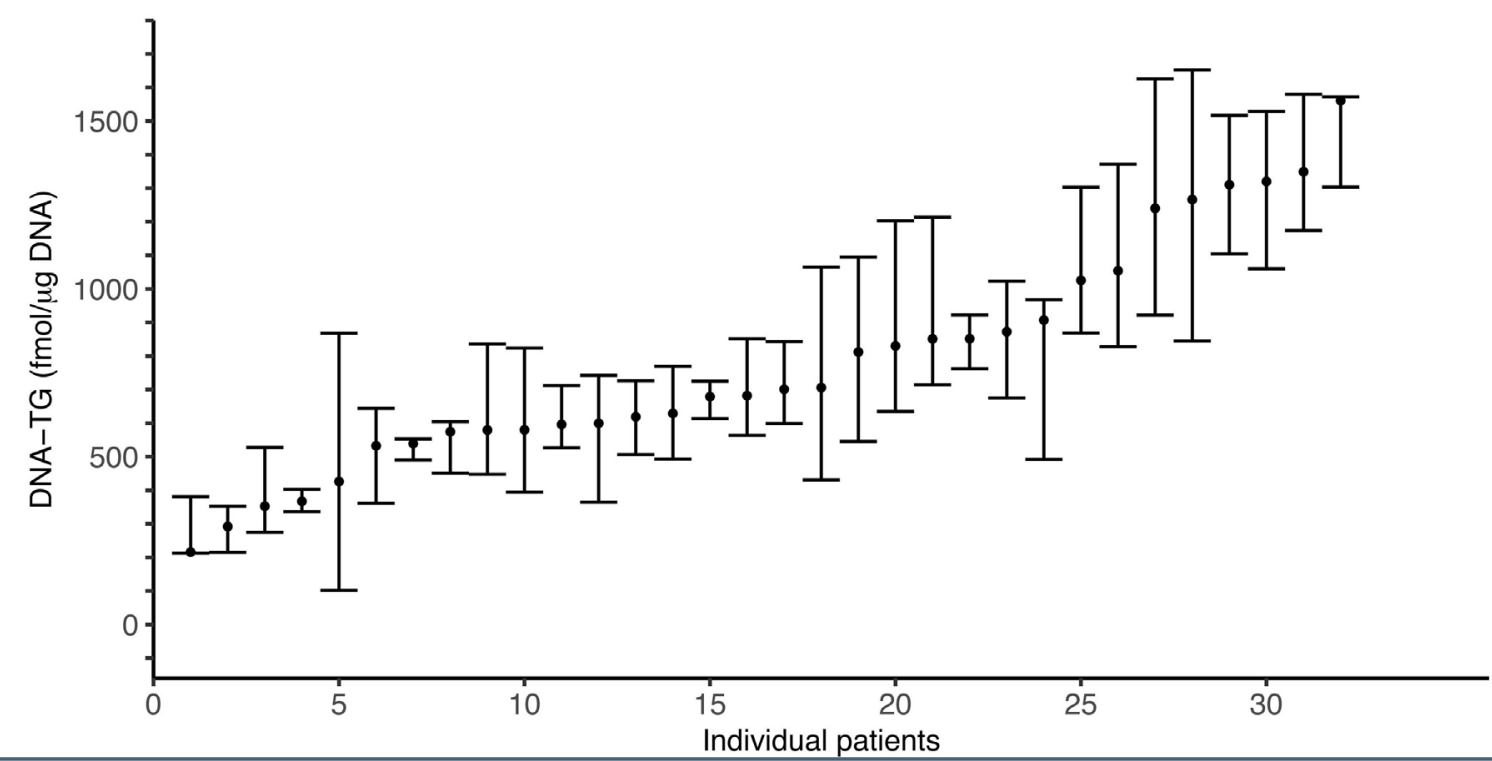

Figure 2. Median DNA-TG from each TEAM patient during TEAM therapy. Median DNA-TG (fmol/ug DNA) and interquartile range for each TEAM patient during TEAM therapy. A total of 32 patients received TEAM maintenance therapy for more than 10 weeks (denoted TEAM therapy). TEAM: thiopurine-enhanced ALL maintenance therapy; DNA-TG: thioguanine nucleotides incorporated into DNA. 
observed. The platelet count was on average $19 \times 10^{9} / \mathrm{L}$ higher $(95 \%$ CI: $3-35 ; P=0.02)$ during TEAM therapy than before TEAM (Figure 4C).

No significant differences were observed, when white blood cell count, absolute neutrophil count and platelet count during TEAM therapy were compared with historical data from the ALL2008 MT substudy $(P=0.09, P=0.07$, and $P=0.18$, respectively) (Tables 2 and 3 ).

\section{Hepatotoxicity}

No hepatic serious adverse events were observed, including no cases of sinusoidal obstruction syndrome. No signif- icant differences were observed when the levels of alanine aminotransferase, coagulation factors II, VII and X, International Normalized Ratio (INR) and bilirubin were compared between the period of TEAM therapy and before TEAM ( $P=0.23, P=0.42, P=0.06$, and $P=0.95$, respectively) (Figure 4D; Tables 2 and 3).

Prior to inclusion in the TEAM protocol, one patient had experienced pronounced symptoms of 6-mercaptopurineinduced hypoglycemia, which disappeared during TEAM therapy. Another patient, prior to TEAM, had experienced recurrent severe hepatoxicity with alanine aminotransferase levels ranging between 522-7,470 U/L (i.e. 166 times

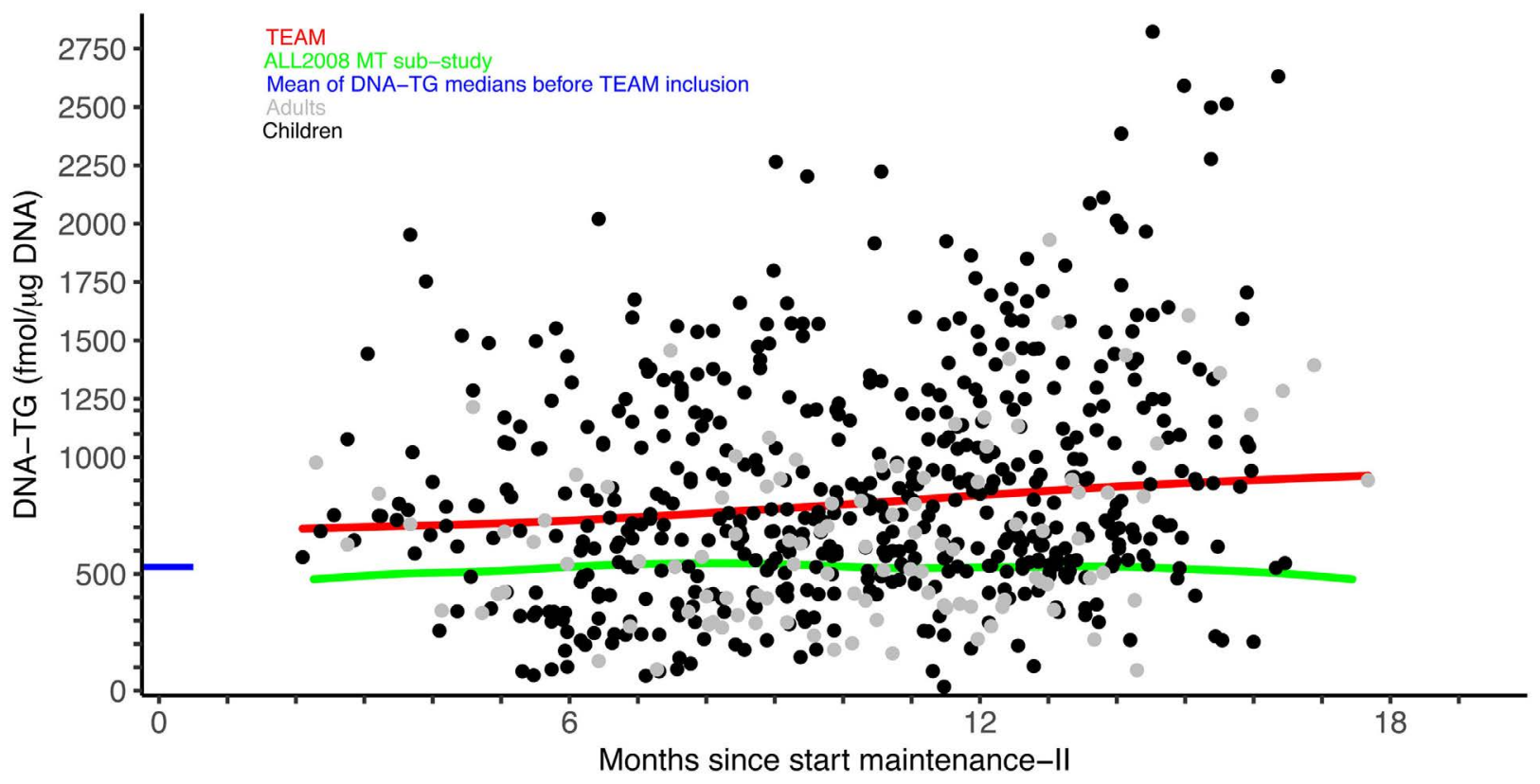

Figure 3. DNA-TG during TEAM therapy. DNA-TG (fmol/ug DNA) during TEAM therapy (red spline function line) compared with DNA-TG levels from the ALL2008 MT substudy (green spline function line), ${ }^{14}$ in which patients received standard methotrexate/6-mercaptopurine maintenance therapy. Each dot refers to one DNA-TG measurement during TEAM therapy (gray dots for adults). The mean of the DNA-TG patients' medians from TEAM patients before their inclusion in TEAM is marked by a blue bar on the Y-axis. TEAM: thiopurine-enhanced ALL maintenance therapy; DNA-TG: thioguanine nucleotides incorporated into DNA.

Table 2. Mean of patients' medians and $95 \%$ reference range for all outcomes.

\begin{tabular}{|c|c|c|c|}
\hline & Before TEAM & TEAM therapy & ALL2008 MT substudy \\
\hline 6-mercaptopurine dose, $\mathrm{mg} / \mathrm{m}^{2} / \mathrm{day}$ & $53(6-121)$ & $45(7-79)$ & - \\
\hline Methotrexate dose, $\mathrm{mg} / \mathrm{m}^{2} /$ week & $19(4-41)$ & $19(5-38)$ & - \\
\hline DNA-TG, fmol/ug & $530(157-1,279)$ & $764(273-1,402)$ & $492(21-1,104)$ \\
\hline Ery-TGN, nmol/mmol hgb & $240(100-485)$ & $721(339-1,396)$ & $231(8-608)$ \\
\hline Ery-MeMP, nmol/mmol hgb & $8,462(177-21,520)$ & $5,931(142-14,385)$ & $12,032(4,577-17,383)^{*}$ \\
\hline White blood cell count, $x 10^{9} / \mathrm{L}$ & $3.1(1.9-5.7)$ & $3.2(2.2-5.5)$ & $2.9(1.7-4.0)$ \\
\hline Absolute neutrophil count, $x 10^{9} / \mathrm{L}$ & $1.8(0.7-3.7)$ & $1.9(1.0-3.7)$ & $1.6(0.5-2.7)$ \\
\hline Platelet count, $x 10^{9} / \mathrm{L}$ & $247(108-371)$ & $261(56-383)$ & $236(117-359)$ \\
\hline Hemoglobin, mmol/L & $7.6(6.3-9.3)$ & $7.7(6.3-9.0)$ & - \\
\hline Alanine aminotransferase, $\mathrm{U} / \mathrm{L}$ & $139(26-373)$ & $118(20-265)$ & - \\
\hline Coagulation factors II-VII-X, IU/L & $0.7(0.4-0.9)$ & $0.7(0.5-0.9)$ & - \\
\hline International Normalized Ratio. & $1.2(1.0-1.6)$ & $1.2(1.1-1.3)$ & - \\
\hline Bilirubin, $\mu \mathrm{mol} / \mathrm{L}$ & $11(4-32)$ & $12(5-29)$ & - \\
\hline
\end{tabular}

Data before TEAM, during TEAM therapy and from the ALL2008 MT substudy (in which patients received standard methotrexate/6-mercaptopurine maintenance therapy ${ }^{14}$ ). Data in this table from "Before TEAM" and from "TEAM therapy" are from the 32 patients comprising the TEAM study population (i.e., patients who received more than 10 weeks of TEAM maintenance therapy). DNA-TG: level of thioguanine nucleotides incorporated into DNA; Ery-TGN: thioguanine nucleotide level in erythrocytes; Ery-MeMP: methylated mercaptopurine metabolite level in erythrocytes; - data not applicable. *Ery-MeMP from the ALL2008 MT substudy is reported as the median of the patients' medians and interquartile range, as medians for this outcome were not normally distributed. 
upper normal level) and coagulation factors II, VII and X $<0.5 \mathrm{IU} / \mathrm{L}$ causing repeated treatment interruptions. During TEAM therapy, no treatment interruptions occurred due to hepatotoxicity.

Four patients developed symptoms of osteonecrosis after inclusion in the TEAM study.

\section{Maximum tolerated 6-thioguanine doses}

There was no apparent dose-response relation between maximum tolerated 6TG dose and median DNA-TG level during TEAM therapy (Figure 5). Of the 30 patients who completed therapy according to the TEAM strategy, 24 tolerated the maximum 6TG dose of $12.5 \mathrm{mg} / \mathrm{m}^{2} / \mathrm{day}$. Two patients tolerated $5 \mathrm{mg} / \mathrm{m}^{2} /$ day, two patients 7.5 $\mathrm{mg} / \mathrm{m}^{2} /$ day, and one patient $10 \mathrm{mg} / \mathrm{m}^{2} /$ day as their maximum 6TG dosage, because higher 6TG doses led to recurring leukopenia/neutropenia. By decision of the treating physician, one patient received $10.0 \mathrm{mg} / \mathrm{m}^{2} /$ day as maximum 6TG dose, as the white blood cell count therapy target was fulfilled with a median DNA-TG of $852 \mathrm{fmol} / \mu \mathrm{g}$ DNA during TEAM therapy, and tolerance of 12.5 $\mathrm{mg} / \mathrm{m}^{2} /$ day 6TG was never tested.

Two patients were heterozygous with one low activity TPMT variant, and both tolerated $12.5 \mathrm{mg} / \mathrm{m}^{2} /$ day $6 \mathrm{TG}$ as their maximum 6TG dosage with no unacceptable toxicities. The median DNA-TG level was $580 \mathrm{fmol} / \mu \mathrm{g}$ DNA and 1,349 fmol/ug DNA during 9 and 16 months of TEAM therapy for these two patients, respectively.

\section{Methotrexate and 6-mercaptopurine doses}

No significant difference was observed when 6-mercaptopurine dose $\left(\mathrm{mg} / \mathrm{m}^{2} /\right.$ day) and methotrexate dose
( $\mathrm{mg} / \mathrm{m}^{2} /$ week) were compared between TEAM therapy and before TEAM, $(P=0.09$ and $P=0.99$, respectively $)$ (Tables 2 and 3 ).

\section{6-Mercaptopurine/methotrexate metabolite levels}

Ery-TGN levels were on average $470 \mathrm{nmol} / \mathrm{mmol}$ hemoglobin higher (95\% CI: 349-590; $P<0.0001)$ during TEAM therapy than before TEAM (Online Supplementary Figure S2A), and on average $490 \mathrm{nmol} / \mathrm{mmol}$ hemoglobin higher (95\% CI: 365-614; $P<0.0001)$ when compared with data from the ALL2008 MT substudy (Tables 2 and 3) A plot of median Ery-TGN levels during TEAM therapy in relation to median DNA-TG level during TEAM therapy is provided in Online Supplementary Figure S3.

Ery-MeMP tended to be lower during TEAM therapy than before TEAM, on average $1,948 \mathrm{nmol} / \mathrm{mmol}$ hemoglobin lower (95\% CI: -4011 to $115, P=0.06$ ), (Online Supplementary Figure S2B). Ery-MeMP was significantly lower during TEAM therapy when compared with data from the ALL2008 MT substudy $(P=0.0001)$ with a difference in the median of patients' levels of 5,363 $\mathrm{nmol} / \mathrm{mmol}$ hemoglobin (Tables 2 and 3 ).

\section{Cessation of TEAM participation}

Of the four patients who discontinued study participation, three discontinued by decision of their parents and/or treating physician, and one patient due to on-therapy leukemic relapse. The background for study discontinuation in one patient was recurring mild hyperbilirubinemia (1.5 times upper normal limit), with no other signs of sinusoidal obstruction syndrome leading to repeated therapy interruptions. The patient's parents
A

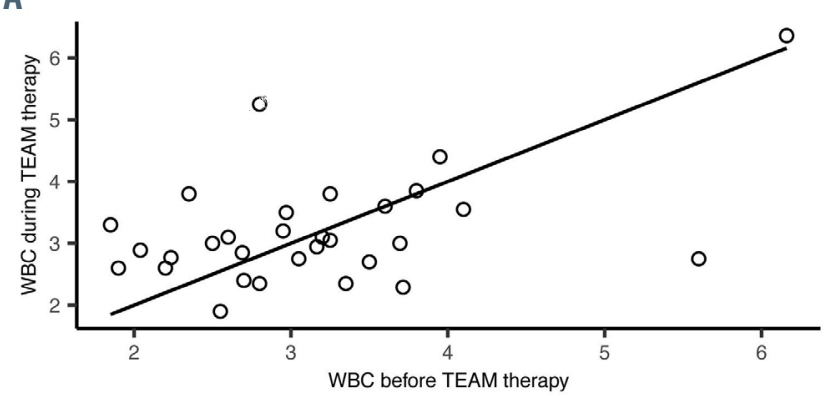

C

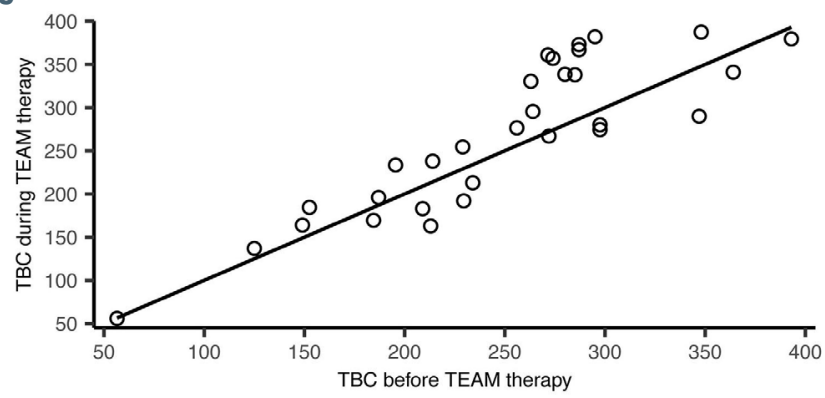

B

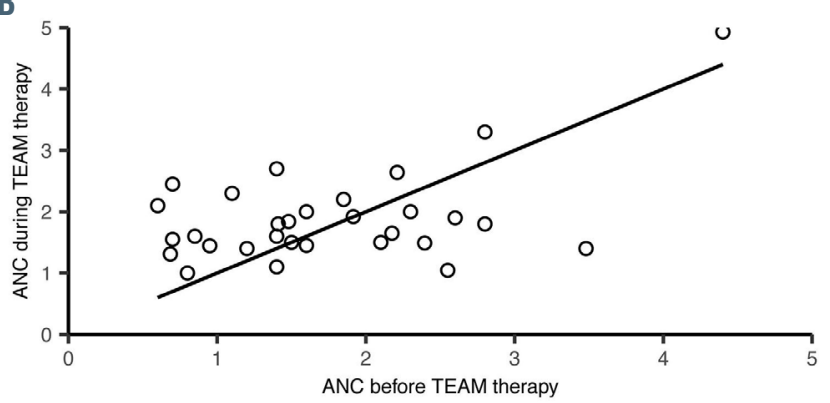

D

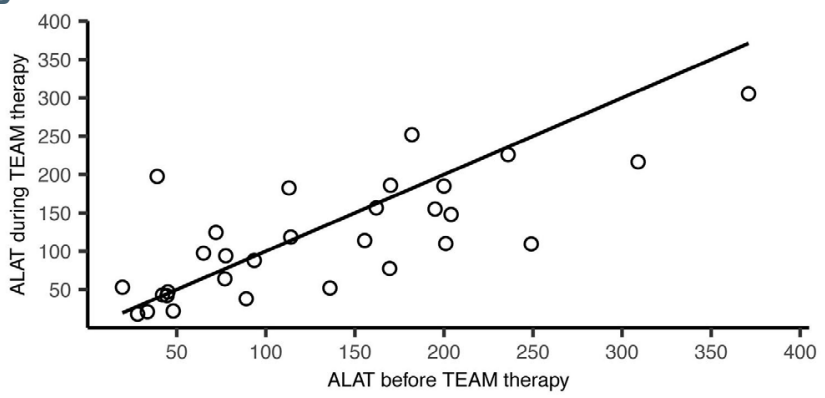

Figure 4. Hematologic and hepatic parameters during TEAM therapy versus before TEAM. (A-D) Hematologic and hepatic parameters during TEAM therapy versus before TEAM. Each circle marks an individual patient's median value during TEAM therapy versus before TEAM. The black line represents the diagonal. (A) Median white blood cell count (x10\% L). (B) Median absolute neutrophil count (x10\% $\mathrm{L}$ ). (C) Median platelet count (x10\% $/ \mathrm{L})$. (D) Median alanine aminotransferase concentration (U/L). TEAM: thiopurine-enhanced ALL maintenance therapy; WBC: white blood cell count; ANC: absolute neutrophil count; TBC: thrombocyte count; ALAT: alanine aminotranscerase. 
decided to opt out of TEAM, and the patient was switched to standard methotrexate/6-mercaptopurine maintenance therapy (during which therapy interruptions are not warranted until bilirubin level exceeds 3 times the upper limit of normal [NOPHO ALL2008 protocol guideline]). The patient continued to demonstrate mild hyperbilirubinemia on conventional methotrexate/6-mercaptopurine maintenance therapy for the remaining 11 months of antileukemic therapy. The background to withdrawal from the study in the second patient was recurring anemia without concurrent leukopenia or thrombocytopenia. When this patient developed symptoms of osteonecrosis the parents took the patient off TEAM. The third patient who discontinued participation in the TEAM study had recurring elevated levels of alanine aminotransferase and P-ferritin reflecting previous multiple red blood cell transfusions. This patient had elevated alanine aminotransferase levels even at the starting 6TG dose of $2.5 \mathrm{mg} / \mathrm{m}^{2} /$ day, but no other signs of liver dysfunction. The patient's parents decided to opt out of TEAM after 2 months, as the elevated liver enzyme levels led to therapy interruptions (in the NOPHO ALL2008

Table 3. Results of comparison of outcomes. TEAM therapy versus before TEAM and TEAM therapy versus data from the ALL2008 MT substudy: mean difference and $95 \%$ confidence interval for all outcomes.

\begin{tabular}{|c|c|c|c|c|}
\hline & \multicolumn{2}{|c|}{$\begin{array}{l}\text { TEAM therapy versus } \\
\text { before TEAM }\end{array}$} & \multicolumn{2}{|c|}{$\begin{array}{l}\text { TEAM therapy versus ALL2008 } \\
\text { MT sub-study }\end{array}$} \\
\hline & & P-value & & P-value \\
\hline 6-mercaptopurine dose, $\mathrm{mg} / \mathrm{m}^{2} /$ day & $-9(-19$ to 1$)$ & $P=0.09 *$ & - & - \\
\hline Methotrexate dose, $\mathrm{mg} / \mathrm{m}^{2} /$ week & $-0.02(-2.8$ to 2.7$)$ & $P=0.99^{*}$ & - & - \\
\hline DNA-TG, fmol/ug & 251 (160 to 341$)$ & $P<0.0001^{*}$ & 272 (147 to 398) & $P<0.0001^{\dagger}$ \\
\hline Ery-TGN, nmol/mmol hb & 470 (349 to 590) & $P<0.0001^{*}$ & 490 (365 to 614) & $P<0.0001^{+}$ \\
\hline Ery-MeMP, nmol/mmol hb & $-1948(-4,011$ to 115$)$ & $P=0.06^{*}$ & - & $P=0.0001^{+}$ \\
\hline White blood cell count, $x 10^{9} / \mathrm{L}$ & $0.05(-0.31$ to 0.41$)$ & $P=0.78^{*}$ & $0.3(-0.05$ to 0.65$)$ & $P=0.09^{\dagger}$ \\
\hline Absolute neutrophil count, $x 10^{9} / \mathrm{L}$ & $0.13(-0.19$ to 0.45$)$ & $P=0.40^{*}$ & $0.3(-0.02$ to 0.57$)$ & $P=0.07^{\dagger}$ \\
\hline Platelet count, $x 10^{9} / \mathrm{L}$ & 19 (2.7 to 35$)$ & $P=0.02 *$ & $24(-12$ to 60$)$ & $P=0.18^{\dagger}$ \\
\hline Hemoglobin, mmol/L & $0.17(-0.04$ to 0.4$)$ & $P=0.10^{*}$ & - & - \\
\hline Alanine aminotransferase, U/L & $-13(-35$ to 9$)$ & $P=0.23^{*}$ & - & - \\
\hline Coagulation factors II-VII-X, IU/L & $0.02(-0.04$ to 0.08$)$ & $P=0.42^{*}$ & - & - \\
\hline International Normalized Ratio & $-0.08(-0.15$ to 0.002$)$ & $P=0.06^{*}$ & - & - \\
\hline Bilirubin, $\mu \mathrm{mol} / \mathrm{L}$ & 0.06 (-1.8 to 1.9$)$ & $P=0.95^{*}$ & - & - \\
\hline
\end{tabular}

Results of comparison of outcomes during TEAM therapy versus before TEAM, and TEAM therapy versus historical data from the ALL2008 MT sub-study (in which patients received standard methotrexate/6-mercaptopurine maintenance therapy ${ }^{14}$ ). Positive values indicate higher values during TEAM therapy, and negative values indicate lower values during TEAM therapy. For comparisons of all outcomes, data from the 32 patients comprising the TEAM study population were used (i.e., from patients who received more than 10 weeks of TEAM maintenance therapy). *Paired $t$-test. 'Two-sample $t$-test. *Wilcoxon signed rank test. DNA-TG: level of thioguanine nucleotides incorporated into DNA; Ery-TGN: thioguanine nucleotide level in erythrocytes; Hb: hemoglobin; Ery-MeMP: methylated mercaptopurine metabolite level in erythrocytes; - data not applicable.

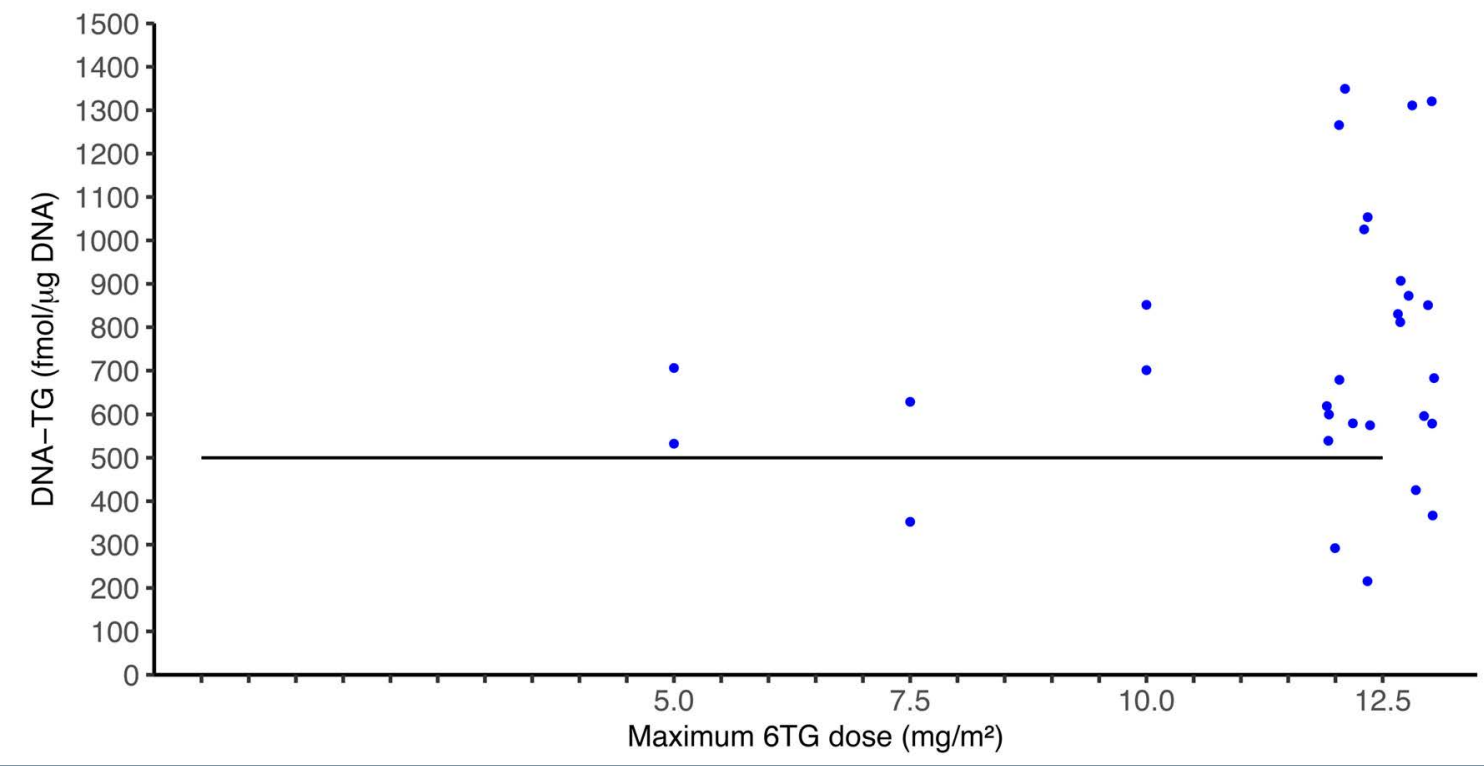

Figure 5. Median DNA-TG during TEAM therapy in relation to 6-thioguanine dose intensity. Median DNA-TG level (fmol/ug DNA) during TEAM therapy from the 30 patients, who completed therapy according to the TEAM strategy (blue dots represent individual patients) in relation to their individual maximum tolerated dose of 6 -thioguanine. The black line indicates the TEAM therapy DNA-TG target of $500 \mathrm{fmol} / \mu \mathrm{g}$ DNA. TEAM: thiopurine-enhanced ALL maintenance therapy; DNA-TG: thioguanine nucleotides incorporated into DNA; 6TG: 6-thioguanine. 
protocol elevated alanine aminotransferase does not in itself warrant therapy interruptions unless accompanied by an INR $>1.5$ or coagulation factors II, VII and X $<0.5$ $\mathrm{IU} / \mathrm{L})$. After discontinuation of $6 \mathrm{TG}$, the patient continued to demonstrate elevated alanine aminotransferase levels on conventional methotrexate/6-mercaptopurine therapy.

Two TEAM patients have been diagnosed with ALL relapse. Both patients had B-cell precursor ALL and were both initially assigned to intermediate-risk therapy due to a poor minimal residual disease response in their end-ofinduction bone-marrow evaluation. The original clone was identified at relapse in both patients. One patient was diagnosed with on-therapy isolated central nervous system relapse, after having received 4 months of TEAM therapy with a median DNA-TG during TEAM therapy of $1,561 \mathrm{fmol} / \mu \mathrm{g}$ DNA. The other patient was diagnosed with isolated bone-marrow relapse 18 months after completion of antileukemic therapy, having received a total of 11 months of TEAM maintenance therapy. Further evaluations of the leukemic clone at relapse revealed an $A B L$ like fusion, which was not identified at initial ALL diagnosis. Re-examination of the leukemic clone from the initial diagnosis confirmed that this aberration had already been present, which could have had an impact on the development of the relapse.

\section{Discussion}

The TEAM strategy was demonstrated to be safe and to result in significantly higher DNA-TG levels compared with those occurring during standard methotrexate/6mercaptopurine maintenance therapy. The estimated reduction in relapse hazard rate of $59 \%$ on the TEAM protocol is theoretical, since the present study was not powered to address survival. However, the increase in DNA-TG on TEAM was significant from both clinical and statistical points of view. The TEAM study therefore introduces a completely novel dosing strategy for methotrexate/thiopurine-based maintenance therapy and could potentially lead to a significant improvement in relapse-free survival through increased DNA-TG. ${ }^{14}$

6TG is more easily converted into thioguanine nucleotides than 6-mercaptopurine and leads to 6- to 7fold higher Ery-TGN levels compared with 6-mercaptopurine at equipotent doses. ${ }^{13,16}$ Furthermore, methotrexate and methylated 6-mercaptopurine metabolites inhibit de novo purine synthesis, and concomitant administration therefore increases DNA-TG formation due to a reduced level of natural guanine to compete with thioguanine nucleotides for incorporation into DNA. ${ }^{11,13,28}$ Based on this synergy of cytotoxic mechanisms, the results of the TEAM study are, even with the very low 6TG dose, well explained. In contrast, attempts to increase DNA-TG by solely incrementing 6-mercaptopurine dose would primarily lead to higher levels of methylated 6-mercaptopurine metabolites rather than thioguanine nucleotides thus shifting cytotoxicity toward inhibition of de novo purine synthesis, but not necessarily higher DNA-TG. ${ }^{13,16}$ Accordingly, 6-mercaptopurine dose during maintenance therapy is not associated with DNA-TG level, ${ }^{14}$ and 6-mercaptopurine dose increments primarily increase the risk of significant hepatotoxicity and may even increase relapse risk. ${ }^{17,18} \mathrm{Of}$ note, the 6TG dose in TEAM did not appear to be associated with median DNA-TG level during TEAM therapy and, as with 6-mercaptopurine, ${ }^{14}$ the DNA-TG level cannot be predicted by 6TG dose intensity.

Upon incorporation of thioguanine nucleotides into DNA, the DNA-TG will undergo random methylation, which will favor mismatching between methyl-thioguanine nucleotides and thymidine. This leads to activation of the mismatch repair system attempting to correct the mismatch. ${ }^{13}$ However, methyl-thioguanine nucleotides will continue to mismatch, which ultimately results in cell death due to repetitive but futile activation of the mismatch repair system. ${ }^{13}$ Tolerance of the increased DNA-TG levels in TEAM most likely reflects the fact that most of the thioguanine nucleotides in DNA are unmethylated and thus do not mismatch.

One of the two patients who was diagnosed with ALL relapse had a median DNA-TG level of 1,561 fmol/ $\mu \mathrm{g}$ DNA during TEAM therapy, which could reflect the presence of an $M S H 6$ deletion causing thiopurine resistance..$^{29}$ However, this was never evaluated, as the patient was referred for hematopoietic stem cell transplantation.

The maximum 6TG dose in the TEAM study was preset at $12.5 \mathrm{mg} / \mathrm{m}^{2} /$ day, as this would correspond to $\sim 75$ $\mathrm{mg} / \mathrm{m}^{2}$ 6-mercatopurine (the standard dose) with respect to Ery-TGN levels. ${ }^{13,16}$ A meta-analysis including three randomized clinical trials comparing methotrexate/6mercaptopurine and methotrexate/6IG based maintenance therapy demonstrated no difference in overall survival between recipients of these two therapy strategies. ${ }^{19-22}$ Although the latter will cause higher cytosolic levels of thioguanine nucleotides, the lack of concomitant inhibition of de novo purine synthesis mediated by methylated 6-mercaptopurine might explain these findings. ${ }^{13,16,19-}$ ${ }^{22}$ However, studies comparing DNA-TG levels during treatment with either 6-mercaptopurine or 6TG as the single thiopurine are lacking.

Methotrexate/6TG-based maintenance therapy has previously been associated with an increased risk of sinusoidal obstruction syndrome. ${ }^{19}$ This syndrome was neither found nor expected among TEAM patients, as a recent systematic review showed that 6TG therapy was not associated with a risk of sinusoidal obstruction syndrome at 6TG doses below $\sim 12.5 \mathrm{mg} / \mathrm{m}^{2} /$ day. $^{30}$

Higher Ery-MeMP has been associated with risk of hepatotoxicity, including a rise in aminotransferase levels $^{18}$ and risk of hypoglycemia. ${ }^{31,32}$ The low Ery-MeMP in TEAM can therefore explain why alanine aminotransferase levels were generally low in the TEAM study, thus highlighting the TEAM strategy as a highly relevant alternative for patients with pronounced 6-mercaptopurine toxicity on conventional methotrexate/6-mercaptopurine maintenance therapy. As an alternative allopurinol has been used to ameliorate this propensity to hepatotoxicity as it shifts patients to a TPMT low-activity phenotype. ${ }^{33}$ However, TPMT heterozygocity is not associated with higher DNA-TG. ${ }^{34}$

In conclusion, DNA-TG level is a composite measure of upstream 6-mercaptopurine, methotrexate; 6TG metabolites and is associated with relapse risk, ${ }^{14}$ and TEAM may be an innovative and feasible approach to improve maintenance therapy by leading to higher DNA-TG levels. TEAM therefore represents a potentially effective strategy for reducing risk of ALL relapse without inducing additional toxicity. This will be tested in a randomized 
ALLTogether-1 substudy (EudraCT number: 2018001795-38).

\section{Disclosures}

KS has received speaker and/or advisory board honoraria from Jazz Pharmaceuticals and Servier; speaker fees from Amgen and Medscape; and an educational grant from Servier. $J K$ has received consulting fees from Bayer. The authors declare that they have no other competing financial interests. The funding and supporting sources listed had no involvement in or restrictions regarding publication.

\section{Contributions}

RHL collected, analyzed, and interpreted data, served as principal investigator for the childhood cases and wrote and edited the manuscript; KS served as sponsor for the TEAM investigation, designed the study, interpreted data, and critically reviewed the manuscript; CUR served as the principal investigator for adults, collected and analyzed data, and critically reviewed the manuscript; LH, BAN, TLF, BKA, PSW, MTC, SNN, HH, and JK served as investigators for childhood cases, collected data and critically reviewed the manuscript; $P K$ and UMO served as investigators for adult cases, collected data and critically reviewed the manuscript; JN and MD performed the analysis and quantification of DNA-TG, Ery-TGN and Ery-MeMP and critically reviewed the manuscript; $L N M$ performed the statistical analyses, created the figures and critically reviewed the manuscript; KG critically reviewed the statistical analyses and the manuscript. All authors approved the final manuscript.

\section{Acknowledgments}

The authors thank the dedicated staff at the laboratory of Pediatric Oncology, Bonkolab, Copenhagen, Denmark for their valuable work with special recognition of the laboratory technicians and project nurses involved.

\section{Funding}

This work was supported by research grants from the Danish Cancer Society, Childhood Cancer Foundation (Denmark), Childhood Cancer Foundation (Sweden), Nordic Cancer Union, Otto Christensen Foundation, The Capital Region of Denmark, and The Research Foundation of Rigshospitalet, University of Copenhagen. This work is part of Childhood Oncology Network Targeting Research, Organization \& Life expectancy (CON$T R O L)$, supported by the Danish Cancer Society (R-257-A14720) and the Danish Childhood Cancer Foundation (2019-5934).

\section{Data-sharing statement}

For original data, please contact Kjeld Schmiegelow: e-mail: kschmiegelow@rh.dk

\section{References}

1. Toft N, Birgens H, Abrahamsson J, et al. Results of NOPHO ALL2008 treatment for patients aged 1-45 years with acute lymphoblastic leukemia. Leukemia. 2018;32(3): 606-615.

2. Hunger SP, Mullighan CG. Acute lymphoblastic leukemia in children. $\mathrm{N}$ Engl J Med. 2015;373(16):1541-1552.

3. Pieters R, de Groot-Kruseman H, Van der Velden V, et al. Successful therapy reduction and intensification for childhood acute lymphoblastic leukemia based on minimal residual disease monitoring: study ALL10 from the Dutch Childhood Oncology Group. J Clin Oncol. 2016;34(22):2591-2601.

4. Dores GM, Devesa SS, Curtis RE, Linet MS, Morton LM. Acute leukemia incidence and patient survival among children and adults in the United States, 2001-2007. Blood. 2012;119(1):34-43.

5. Malempati S, Gaynon PS, Sather H, La MK, Stork LC. Outcome after relapse among children with standard-risk acute lymphoblastic leukemia: Children's Oncology Group study CCG-1952. J Clin Oncol. 2007;25(36):58005807.

6. Oskarsson T, Soderhall S, Arvidson J, et al. Relapsed childhood acute lymphoblastic leukemia in the Nordic countries: prognostic factors, treatment and outcome. Haematologica. 2016;101(1):68-76.

7. Schmiegelow K, Nielsen SN, Frandsen TL, Nersting J. Mercaptopurine/methotrexate maintenance therapy of childhood acute lymphoblastic leukemia: clinical facts and fiction. J Pediatr Hematol Oncol. 2014;36(7): 503-517.

8. Arico M, Baruchel A, Bertrand Y, et al. The seventh international childhood acute lymphoblastic leukemia workshop report: Palermo, Italy, January 29-30, 2005. Leukemia. 2005;19(7):1145-1152.

9. Schmiegelow K, Nersting J, Nielsen SN, et al. Maintenance therapy of childhood acute lymphoblastic leukemia revisited - should drug doses be adjusted by white blood cell, neutrophil, or lymphocyte counts? Pediatr Blood Cancer. 2016;63(12):2104-2111.

10. Haus E, Smolensky MH. Biologic rhythms in the immune system. Chronobiol Int. 1999;16(5):581-622.

11. Nielsen SN, Grell K, Nersting J, Frandsen TL, Hjalgrim LL, Schmiegelow K. Measures of 6mercaptopurine and methotrexate maintenance therapy intensity in childhood acute lymphoblastic leukemia. Cancer Chemother Pharmacol. 2016;78(5):983-994.

12. Haddy TB, Rana SR, Castro O. Benign ethnic neutropenia: what is a normal absolute neutrophil count? J Lab Clin Med. 1999;133(1): 15-22.

13. Karran P, Attard N. Thiopurines in current medical practice: molecular mechanisms and contributions to therapy-related cancer. Nat Rev Cancer. 2008;8(1):24-36.

14. Nielsen SN, Grell K, Nersting J, et al. DNAthioguanine nucleotide concentration and relapse-free survival during maintenance therapy of childhood acute lymphoblastic leukaemia (NOPHO ALL2008): a prospective substudy of a phase 3 trial. Lancet Oncol. 2017;18(4):515-524.

15. Schmiegelow K, Forestier E, Kristinsson J, et al. Thiopurine methyltransferase activity is related to the risk of relapse of childhood acute lymphoblastic leukemia: results from the NOPHO ALL-92 study. Leukemia. 2009;23(3):557-564

16. Erb N, Harms DO, Janka-Schaub G. Pharmacokinetics and metabolism of thiopurines in children with acute lymphoblastic leukemia receiving 6-thioguanine versus 6mercaptopurine. Cancer Chemother Pharmacol. 1998;42(4):266-272.

17. Schmiegelow K, Bjork O, Glomstein A, et al. Intensification of mercaptopurine/ methotrexate maintenance chemotherapy may increase the risk of relapse for some children with acute lymphoblastic leukemia. J Clin Oncol. 2003;21(7):1332-1339.

18. Nygaard U, Toft N, Schmiegelow K.
Methylated metabolites of 6-mercaptopurine are associated with hepatotoxicity. Clin Pharmacol Ther. 2004;75(4):274-281.

19. Escherich G, Richards S, Stork LC, Vora AJ Meta-analysis of randomised trials comparing thiopurines in childhood acute lymphoblastic leukaemia. Leukemia. 2011;25(6): 953-959.

20. Vora A, Mitchell CD, Lennard L, et al Toxicity and efficacy of 6-thioguanine versus 6-mercaptopurine in childhood lymphoblastic leukaemia: a randomised trial. Lancet. 2006;368(9544):1339-1348.

21. Harms DO, Gobel U, Spaar HJ, et al. Thioguanine offers no advantage over mercaptopurine in maintenance treatment of childhood ALL: results of the randomized trial COALL-92. Blood. 2003;102(8):27362740 .

22. Stork LC, Matloub Y, Broxson E, et al. Oral 6-mercaptopurine versus oral 6-thioguanine and veno-occlusive disease in children with standard-risk acute lymphoblastic leukemia: report of the Children's Oncology Group CCG-1952 clinical trial. Blood. 2010;115 (14):2740-2748.

23. Toft N, Birgens H, Abrahamsson J, et al. Risk group assignment differs for children and adults 1-45 yr with acute lymphoblastic leukemia treated by the NOPHO ALL-2008 protocol. Eur J Haematol. 2013;90(5):404412.

24. Frandsen TL, Heyman M, Abrahamsson J, et al. Complying with the European Clinical Trials directive while surviving the administrative pressure - an alternative approach to toxicity registration in a cancer trial. Eur J Cancer. 2014;50(2):251-259.

25. Schmiegelow K, Attarbaschi A, Barzilai S, et al. Consensus definitions of 14 severe acute toxic effects for childhood lymphoblastic leukaemia treatment: a Delphi consensus. Lancet Oncol. 201617(6):e231-e239.

26. Jacobsen JH, Schmiegelow K, Nersting J. Liquid chromatography-tandem mass spectrometry quantification of 6-thioguanine in DNA using endogenous guanine as internal 
standard. J Chromatogr B Analyt Technol Biomed Life Sci. 2012;881-882:115-118.

27. Shipkova M, Armstrong VW, Wieland E, Oellerich M. Differences in nucleotide hydrolysis contribute to the differences between erythrocyte 6-thioguanine nucleotide concentrations determined by two widely used methods. Clin Chem. 2003;49(2):260-268.

28. Chabner BA, Allegra CJ, Curt GA, et al. Polyglutamation of methotrexate. Is methotrexate a prodrug? J Clin Invest. 1985;76(3):907-912.

29. Evensen NA, Madhusoodhan PP, Meyer J, et al. MSH6 haploinsufficiency at relapse contributes to the development of thiopurine resistance in pediatric B-lymphoblastic leukemia. Haematologica. 2018;103(5):830839.

30. Toksvang LN, Schmidt MS, Arup S, et al. Hepatotoxicity during 6-thioguanine treatment in inflammatory bowel disease and childhood acute lymphoblastic leukaemia: a systematic review. PLoS One. 2019;14(5): e0212157.

31. Halonen P, Salo MK, Makipernaa A. Fasting hypoglycemia is common during maintenance therapy for childhood acute lymphoblastic leukemia. J Pediatr. 2001;138(3): 428-431.

32. Melachuri S, Gandrud L, Bostrom B. The association between fasting hypoglycemia and methylated mercaptopurine metabolites in children with acute lymphoblastic leukemia. Pediatr Blood Cancer. 2014;61(6): 1003-1006.

33. Cohen G, Cooper S, Sison EA, Annesley C, Bhuiyan M, Brown P. Allopurinol use during pediatric acute lymphoblastic leukemia maintenance therapy safely corrects skewed 6-mercaptopurine metabolism, improving inadequate myelosuppression and reducing gastrointestinal toxicity. Pediatr Blood Cancer. 2020;67(11):e28360.

34. Ebbesen MS, Nersting J, Jacobsen JH, et al Incorporation of 6-thioguanine nucleotides into DNA during maintenance therapy of childhood acute lymphoblastic leukemiathe influence of thiopurine methyltransferase genotypes. J Clin Pharmacol. 2013;53 (6):670-674 